\title{
On Composition Ideals of Multilinear Mappings and Homogeneous Polynomials
}

\author{
By \\ Geraldo Botelho*, Daniel Pellegrino** and Pilar RuedA***
}

\begin{abstract}
Given an operator ideal $\mathcal{I}$, we study the multi-ideal $\mathcal{I} \circ \mathcal{L}$ and the polynomial ideal $\mathcal{I} \circ \mathcal{P}$. The connection with the linearizations of these mappings on projective symmetric tensor products is investigated in detail. Applications to the ideals of strictly singular and absolutely summing linear operators are obtained.
\end{abstract}

\section{$\S 1$. Introduction}

Since the 1983 paper by A. Pietsch [26], ideals of multilinear mappings (multi-ideals) and homogeneous polynomials (polynomial ideals) between Banach spaces have been studied as a natural consequence of the successful theory of operator ideals. Several ideals have been investigated and abstract methods to generate ideals of multilinear mappings and polynomials have been introduced (see $[6,20])$.

A multilinear mapping $A$ between Banach spaces is compact (weakly compact) if and only if $A$ can be written as $A=u \circ B$ where $B$ is a multilinear

Communicated by H. Okamoto. Received February 6, 2007. Revised June 11, 2007.

2000 Mathematics Subject Classification(s): Primary 46G25; Secondary 46B28, 47B10.

*Faculdade de Matemática, Universidade Federal de Uberlândia, 38.400-902 - Uberlândia, Brazil.

e-mail: botelho@ufu.br

Supported by CNPq Project 202162/2006-0.

** Departamento de Matemática, Universidade Federal da Paraíba, 58.051-900 - João Pessoa, Brazil.

e-mail: dmpellegrino@gmail.com

Supported by CNPq Projects 471054/2006-2 and 308084/2006-3.

*** Departamento de Análisis Matemático, Universidad de Valencia, 46.100 Burjasot - Valencia, Spain.

e-mail: pilar.rueda@uv.es

Supported by MEC and FEDER Project MTM2005-08210.

(c) 2007 Research Institute for Mathematical Sciences, Kyoto University. All rights reserved. 
mapping and $u$ is a compact (weakly compact) linear operator. A similar characterization holds for compact and weakly compact homogeneous polynomials (see $[25,27])$. So, given an operator ideal $\mathcal{I}$, it is natural to consider the multilinear mappings $A$ and the polynomials $P$ which can be written as $A=u \circ B$ and $P=u \circ Q$ with $u$ belonging to $\mathcal{I}$. This is a particular case of the technique known as composition ideals (see [20, 7.3]). In Section 2 we investigate the resulting multi and polynomial (normed, Banach) ideals exploring the connection with the linearizations of such mappings on the projective tensor product.

In Section 3 we consider the ideal of strictly singular linear operators, an ideal which gained much importance with the Gowers-Maurey theory of hereditarily indecomposable spaces. We provide examples, counterexamples and prove some properties of the composition polynomial ideal generated by the ideal of strictly singular operators.

In Section 4 we relate the composition multi-ideal generated by the ideal of absolutely summing linear operators with some other well studied classes. As consequences, a question raised by R. Alencar-M. C. Matos [2] is solved and an application to dominated polynomials on $C(K)$-spaces is obtained.

\section{$\S 2 . \quad$ Background and Notation}

Throughout this paper $E_{1}, \ldots, E_{n}, E, F, G, G_{1}, \ldots, G_{n}$ will stand for Banach spaces over $\mathbb{K}=\mathbb{R}$ or $\mathbb{C}$ and $n$ will always be a positive integer. By $\mathcal{L}\left(E_{1}, \ldots, E_{n} ; F\right)$ and $\mathcal{P}\left({ }^{n} E ; F\right)$ we denote the Banach spaces of all continuous $n$-linear mappings from $E_{1} \times \cdots \times E_{n}$ to $F$ and continuous $n$-homogeneous polynomials from $E$ to $F$, respectively, both of them with the usual sup norm. If $E_{1}=\cdots=E_{n}=E$ we write $\mathcal{L}\left({ }^{n} E ; F\right)$. If $F=\mathbb{K}$ we simply write $\mathcal{L}\left(E_{1}, \ldots, E_{n}\right), \mathcal{L}\left({ }^{n} E\right)$ and $\mathcal{P}\left({ }^{n} E\right)$. Given $P \in \mathcal{P}\left({ }^{n} E ; F\right)$, by $\check{P}$ we mean the continuous symmetric $n$-linear mapping associated to the polynomial $P$. By $A_{S}$ we denote the symmetrization of the multilinear mapping $A$ and by $\hat{A}$ we mean the polynomial generated by $A$, that is $\hat{A}(x)=A(x, \ldots, x)$. The $n$-th polarization constant of the Banach space $E$ is denoted by $c(n, E)$, that is,

$$
c(n, E)=\inf \left\{C>0:\|\check{P}\| \leq C\|P\| \text {, for all } P \in \mathcal{P}\left({ }^{n} E\right)\right\} .
$$

For the general theory of multilinear mappings and homogeneous polynomials we refer to S. Dineen [18].

By $E_{1} \widehat{\otimes}_{\pi} \cdots \widehat{\otimes}_{\pi} E_{n}$ we denote the completed projective tensor product of $E_{1}, \ldots, E_{n}$. If $E_{1}=\cdots=E_{n}=E$ we write $\widehat{\otimes}_{\pi}^{n} E$. For the theory of topological tensor products we refer to R. Ryan [28]. By $\widehat{\otimes}_{\pi}^{n, s} E$ and $\widehat{\otimes}_{\pi_{s}}^{n, s} E$ we denote the $n$-fold completed symmetric tensor product of $E$ endowed with the projective 
norm $\pi$ and the projective $s$-tensor norm $\pi_{s}$, respectively. The projective norm $\pi$ is well-known and the projective $s$-tensor norm $\pi_{s}$ is defined by

$$
\pi_{s}(z)=\inf \left\{\sum_{j=1}^{k}\left|\lambda_{j}\right|\left\|x_{j}\right\|^{n}: k \in \mathbb{N}, z=\sum_{j=1}^{k} \lambda_{j} x_{j} \otimes \cdots \otimes x_{j}\right\}
$$

for $z \in \otimes^{n, s} E$. For the properties of $\pi_{s}$ and the general theory of symmetric tensor products we refer to K. Floret [19].

An ideal of multilinear mappings (or multi-ideal) $\mathcal{M}$ is a subclass of the class of all continuous multilinear mappings between Banach spaces such that for all $n \in \mathbb{N}$ and Banach spaces $E_{1}, \ldots, E_{n}$ and $F$, the components $\mathcal{M}\left(E_{1}, \ldots\right.$, $\left.E_{n}, F\right):=\mathcal{L}\left(E_{1}, \ldots, E_{n}, F\right) \cap \mathcal{M}$ satisfy:

(i) $\mathcal{M}\left(E_{1}, \ldots, E_{n}, F\right)$ is a linear subspace of $\mathcal{L}\left(E_{1}, \ldots, E_{n}, F\right)$ which contains the $n$-linear mappings of finite type.

(ii) The ideal property: if $A \in \mathcal{M}\left(E_{1}, \ldots, E_{n}, F\right), u_{j} \in \mathcal{L}\left(G_{j}, E_{j}\right)$ for $j=$ $1, \ldots, n$ and $t \in \mathcal{L}(F, H)$, then $t \circ A \circ\left(u_{1}, \ldots, u_{n}\right)$ is in $\mathcal{M}\left(G_{1}, \ldots, G_{n}, H\right)$.

If $\|\cdot\|_{\mathcal{M}}: \mathcal{M} \rightarrow \mathbb{R}^{+}$satisfies

(i') $\left(\mathcal{M}\left(E_{1}, \ldots, E_{n} ; F\right),\|\cdot\|_{\mathcal{M}}\right)$ is a normed (Banach) space for all Banach spaces $E_{1}, \ldots, E_{n}$ and $F$ and all $n$,

(ii') $\left\|A^{n}: \mathbb{K}^{n} \rightarrow \mathbb{K}: A^{n}\left(x_{1}, \ldots, x_{n}\right)=x_{1} \cdots x_{n}\right\|_{\mathcal{M}}=1$ for all $n$,

(iii') If $A \in \mathcal{M}\left(E_{1}, \ldots, E_{n}, F\right), u_{j} \in \mathcal{L}\left(G_{j}, E_{j}\right)$ for $j=1, \ldots, n$ and $t \in \mathcal{L}(F, H)$, then $\left\|t \circ A \circ\left(u_{1}, \ldots, u_{n}\right)\right\|_{\mathcal{M}} \leq\|t\|\|A\|_{\mathcal{M}}\left\|u_{1}\right\| \cdots\left\|u_{n}\right\|$,

then $\left(\mathcal{M},\|\cdot\|_{\mathcal{M}}\right)$ is called a normed (Banach) multi-ideal.

The multi-ideal $\mathcal{M}$ is said to be

- closed if each $\mathcal{M}\left(E_{1}, \ldots, E_{n}, F\right)$ is a closed subspace of $\mathcal{L}\left(E_{1}, \ldots, E_{n}, F\right)$;

- symmetric (cf. $[9,21]$ ) if $A_{S} \in \mathcal{M}$ whenever $A \in \mathcal{M}$.

An ideal of homogeneous polynomials (or polynomial ideal) $\mathcal{Q}$ is a subclass of the class of all continuous homogeneous polynomials between Banach spaces such that for all $n \in \mathbb{N}$ and Banach spaces $E$ and $F$, the components $\mathcal{Q}\left({ }^{n} E, F\right)=\mathcal{P}\left({ }^{n} E, F\right) \cap \mathcal{Q}$ satisfy:

(i) $\mathcal{Q}\left({ }^{n} E, F\right)$ is a linear subspace of $\mathcal{P}\left({ }^{n} E, F\right)$ which contains the $n$-homogeneous polynomials of finite type.

(ii) The ideal property: if $u \in \mathcal{L}(G, E), P \in \mathcal{Q}\left({ }^{n} E, F\right)$ and $t \in \mathcal{L}(F, H)$, then the composition $t \circ P \circ u$ is in $\mathcal{Q}\left({ }^{n} G, H\right)$.

If $\|\cdot\|_{\mathcal{Q}}: \mathcal{Q} \rightarrow \mathbb{R}^{+}$satisfies

(i') $\left(\mathcal{Q}\left({ }^{n} E ; F\right),\|\cdot\|_{\mathcal{Q}}\right)$ is a normed (Banach) space for all $E, F$ and $n$,

(ii') $\left\|P^{n}: \mathbb{K} \rightarrow \mathbb{K}: P^{n}(x)=x^{n}\right\|_{\mathcal{Q}}=1$ for all $n$,

(iii') If $u \in \mathcal{L}(G, E), P \in \mathcal{Q}\left({ }^{n} E, F\right)$ and $t \in \mathcal{L}(F, H)$, then $\|t \circ P \circ u\|_{\mathcal{Q}} \leq$ $\|t\|\|P\|_{\mathcal{Q}}\|u\|^{n}$, 
then $\left(\mathcal{Q},\|\cdot\|_{\mathcal{Q}}\right)$ is called a normed (Banach) polynomial ideal.

The polynomial ideal $\mathcal{Q}$ is said to be closed if each component $\mathcal{Q}\left({ }^{n} E ; F\right)$ is a closed subspace of $\mathcal{P}\left({ }^{n} E ; F\right)$.

The case $n=1$ recovers the classical theory of (normed, Banach) operator ideals, for which the reader is referred to [15].

\section{$\S 3 . \quad$ Composition Ideals}

Actually, we consider only a particular case of the procedure called composition ideals to generate multi and polynomials ideals from a given operator ideal.

Definition 3.1. Let $\mathcal{I}$ be an operator ideal.

(a) An $n$-linear mapping $A \in \mathcal{L}\left(E_{1}, \ldots, E_{n} ; F\right)$ belongs to $\mathcal{I} \circ \mathcal{L}$ - in this case we write $A \in \mathcal{I} \circ \mathcal{L}\left(E_{1}, \ldots, E_{n} ; F\right)$ - if there are a Banach space $G$, an $n$ linear mapping $B \in \mathcal{L}\left(E_{1}, \ldots, E_{n} ; G\right)$ and an operator $u \in \mathcal{I}(G ; F)$ such that $A=u \circ B$.

(b) An $n$-homogeneous polynomial $P \in \mathcal{P}\left({ }^{n} E ; F\right)$ belongs to $\mathcal{I} \circ \mathcal{P}$ - in this case we write $P \in \mathcal{I} \circ \mathcal{P}\left({ }^{n} E ; F\right)$ - if there are a Banach space $G$, an $n$-homogeneous polynomial $Q \in \mathcal{P}\left({ }^{n} E ; G\right)$ and an operator $u \in \mathcal{I}(G ; F)$ such that $P=u \circ Q$.

It is obvious that continuous multilinear forms belong to $\mathcal{I} \circ \mathcal{L}$ and continuous scalar-valued homogeneous polynomials belong to $\mathcal{I} \circ \mathcal{P}$.

Given $A \in \mathcal{L}\left(E_{1}, \ldots, E_{n} ; F\right)$ and $P \in \mathcal{P}\left({ }^{n} E ; F\right)$ consider their linearizations

$$
\begin{gathered}
A_{L}: E_{1} \widehat{\otimes}_{\pi} \cdots \widehat{\otimes}_{\pi} E_{n} \longrightarrow F, A_{L}\left(x_{1} \otimes \cdots \otimes x_{n}\right)=A\left(x_{1}, \ldots, x_{n}\right) ; \\
P_{L}: \widehat{\otimes}_{\pi}^{n, s} E \longrightarrow F, P_{L}(x \otimes \cdots \otimes x)=P(x) ; \\
P_{L, s}: \widehat{\otimes}_{\pi_{s}}^{n, s} E \longrightarrow F, P_{L, s}(x \otimes \cdots \otimes x)=P(x) .
\end{gathered}
$$

It is well known that $\left\|A_{L}\right\|=\|A\|,\left\|P_{L, s}\right\|=\|P\|$ and $\left\|P_{L}\right\|=\|\check{P}\|$ (see [19]).

Proposition 3.2. $\quad$ Let $\mathcal{I}$ be an operator ideal.

(a) The following are equivalent for $A \in \mathcal{L}\left(E_{1}, \ldots, E_{n} ; F\right)$ :

(a1) $A \in \mathcal{I} \circ \mathcal{L}\left(E_{1}, \ldots, E_{n} ; F\right)$.

(a2) $A_{L} \in \mathcal{I}\left(E_{1} \widehat{\otimes}_{\pi} \cdots \widehat{\otimes}_{\pi} E_{n} ; F\right)$.

(b) The following are equivalent for $P \in \mathcal{P}\left({ }^{n} E ; F\right)$ :

(b1) $P \in \mathcal{I} \circ \mathcal{P}\left({ }^{n} E ; F\right)$.

(b2) $P_{L} \in \mathcal{I}\left(\widehat{\otimes}_{\pi}^{n, s} E ; F\right)$. 
(b3) $P_{L, s} \in \mathcal{I}\left(\widehat{\otimes}_{\pi_{s}}^{n, s} E ; F\right)$.

(b4) $\check{P} \in \mathcal{I} \circ \mathcal{L}\left({ }^{n} E ; F\right)$.

(b5) There is $A \in \mathcal{I} \circ \mathcal{L}\left({ }^{n} E ; F\right)$ such that $\hat{A}=P$.

Proof. $\quad($ a1 $) \Rightarrow\left(\right.$ a2) Let $A=u \circ B$ with $B \in \mathcal{L}\left(E_{1}, \ldots, E_{n} ; G\right)$ and $u \in$ $\mathcal{I}(G ; F)$. For every $x_{j} \in E_{j}, j=1, \ldots, n$,

$$
\begin{aligned}
\left(u \circ B_{L}\right)\left(x_{1} \otimes \cdots \otimes x_{n}\right) & =u\left(B_{L}\left(x_{1} \otimes \cdots \otimes x_{n}\right)\right)=u\left(B\left(x_{1}, \ldots, x_{n}\right)\right) \\
& =A\left(x_{1}, \ldots, x_{n}\right)=A_{L}\left(x_{1} \otimes \cdots \otimes x_{n}\right),
\end{aligned}
$$

showing that $A_{L}=u \circ B_{L}$ (remember that both $A_{L}$ and $u \circ B_{L}$ are linear). So $A_{L} \in \mathcal{I}\left(E_{1} \widehat{\otimes}_{\pi} \cdots \widehat{\otimes}_{\pi} E_{n} ; F\right)$ by the ideal property.

(a2) $\Rightarrow$ (a1) Let $\sigma_{n}: E_{1} \times \cdots \times E_{n} \longrightarrow E_{1} \widehat{\otimes}_{\pi} \cdots \widehat{\otimes}_{\pi} E_{n}$ be the canonical $n$-linear mapping given by $\sigma_{n}\left(x_{1}, \ldots, x_{n}\right)=x_{1} \otimes \cdots \otimes x_{n}$. The factorization $A=A_{L} \circ \sigma_{n}$ shows that $A \in \mathcal{I} \circ \mathcal{L}\left(E_{1}, \ldots, E_{n} ; F\right)$.

(b1) $\Rightarrow$ (b2) As in the proof of (a1) $\Rightarrow(\mathrm{a} 2), P_{L}=u \circ Q_{L}$ if $P=u \circ Q$.

(b2) $\Rightarrow$ (b1) Consider the canonical polynomial

$$
\delta_{n}: E \longrightarrow \widehat{\otimes}_{\pi}^{n, s} E: \delta_{n}(x)=x \otimes \cdots \otimes x ; \delta_{n} \in \mathcal{P}\left({ }^{n} E ; \widehat{\otimes}_{\pi}^{n, s} E\right) .
$$

The factorization $P=P_{L} \circ \delta_{n}$ shows that $P \in \mathcal{I} \circ \mathcal{P}\left({ }^{n} E ; F\right)$.

(b2) $\Leftrightarrow$ (b3) This equivalence follows from the inequalities $\pi \leq \pi_{s} \leq c(n, E) \pi$, which hold on $\otimes^{n, s} E$ (see [19, p. 162]).

(b2) $\Rightarrow$ (b4) Let $S_{n}: \widehat{\otimes}_{\pi}^{n} E \longrightarrow \widehat{\otimes}_{\pi}^{n, s} E$ be the symmetrization operator, that is

$$
S_{n}\left(x_{1} \otimes \cdots \otimes x_{n}\right)=\frac{1}{n !} \sum_{\tau \in \Delta_{n}} x_{\tau(1)} \otimes \cdots \otimes x_{\tau(n)},
$$

where $\Delta_{n}$ is the set of all permutations of $\{1, \ldots, n\}$. For every $x_{1}, \ldots, x_{n} \in E$, using that $\check{P}_{L}$ and $P_{L}$ coincide on $\widehat{\otimes}_{\pi}^{n, s} E$ and that $\check{P}$ is symmetric we obtain

$$
\begin{aligned}
\left(P_{L} \circ S_{n} \circ \sigma_{n}\right)\left(x_{1}, \ldots, x_{n}\right) & =P_{L}\left(S_{n}\left(\sigma_{n}\left(x_{1}, \ldots, x_{n}\right)\right)\right)=P_{L}\left(S_{n}\left(x_{1} \otimes \cdots \otimes x_{n}\right)\right) \\
& =P_{L}\left(\frac{1}{n !} \sum_{\tau \in \Delta_{n}} x_{\tau(1)} \otimes \cdots \otimes x_{\tau(n)}\right) \\
& =\check{P}_{L}\left(\frac{1}{n !} \sum_{\tau \in \Delta_{n}} x_{\tau(1)} \otimes \cdots \otimes x_{\tau(n)}\right) \\
& =\frac{1}{n !} \sum_{\tau \in \Delta_{n}} \check{P}_{L}\left(x_{\tau(1)} \otimes \cdots \otimes x_{\tau(n)}\right) \\
& =\frac{1}{n !} \sum_{\tau \in \Delta_{n}} \check{P}\left(x_{\tau(1)}, \ldots, x_{\tau(n)}\right)=\check{P}\left(x_{1}, \ldots, x_{n}\right)
\end{aligned}
$$


showing that $\check{P}=P_{L} \circ S_{n} \circ \sigma_{n} . P_{L} \in \mathcal{I}\left(\widehat{\otimes}_{\pi}^{n, s} E ; F\right)$ by assumption, so $P_{L} \circ S_{n} \in$ $\mathcal{I}\left(\widehat{\otimes}_{\pi}^{n} E ; F\right)$. The factorization $\check{P}=\left(P_{L} \circ S_{n}\right) \circ \sigma_{n}$ yields that $\check{P} \in \mathcal{I} \circ \mathcal{L}\left({ }^{n} E ; F\right)$. (b4) $\Rightarrow$ (b5) This is obvious with $A=\check{P}$.

(b5) $\Rightarrow(\mathrm{b} 1)$ There is $u$ in $\mathcal{I}$ such that $A=u \circ B$. So $P=\hat{A}=u \circ \hat{B}$.

Proposition 3.3. Let $\mathcal{I}$ be a (closed) operator ideal. Then:

(a) $\mathcal{I} \circ \mathcal{L}$ is a symmetric (closed) multi-ideal.

(b) $\mathcal{I} \circ \mathcal{P}$ is a (closed) polynomial ideal.

Proof. That $\mathcal{I} \circ \mathcal{L}$ and $\mathcal{I} \circ \mathcal{P}$ are (closed) ideals is folklore. Let us prove that $\mathcal{I} \circ \mathcal{L}$ is symmetric: given $A \in \mathcal{I} \circ \mathcal{L}\left({ }^{n} E ; F\right)$, by Proposition 3.2 ((b5) $\Rightarrow$ (b1)) we know that $\hat{A} \in \mathcal{I} \circ \mathcal{P}\left({ }^{n} E ; F\right)$, so $A_{S}=(\hat{A})^{\vee} \in \mathcal{I} \circ \mathcal{L}\left({ }^{n} E ; F\right)$ by $((\mathrm{b} 1)$ $\Rightarrow(\mathrm{b} 4))$ of the same proposition.

Let $\mathcal{K}$ and $\mathcal{W}$ be the closed operator ideals formed by all compact and weakly compact linear operators, respectively. By $\mathcal{P}_{\mathcal{K}}$ and $\mathcal{P}_{\mathcal{W}}$ we mean the classes of all compact and weakly compact polynomials, respectively. The equalities $\mathcal{P}_{\mathcal{K}}=\mathcal{K} \circ \mathcal{P}$ and $\mathcal{P}_{\mathcal{W}}=\mathcal{W} \circ \mathcal{P}$ were proved by R. Ryan [27] (their multilinear analogues were proved by A. Pełczyński [25]). The equivalences $P \in \mathcal{K} \circ \mathcal{P} \Longleftrightarrow \check{P} \in \mathcal{K} \circ \mathcal{L}$ and $P \in \mathcal{W} \circ \mathcal{P} \Longleftrightarrow \check{P} \in \mathcal{W} \circ \mathcal{L}$ follow from a combination of [25, Proposition 3] and [28, Lemma 4.1]. Our results extend this fact to arbitrary operator ideals.

Next we show that $\mathcal{I} \circ \mathcal{L}$ and $\mathcal{I} \circ \mathcal{P}$ extend typical linear behavior to the nonlinear context. The identity operator on a Banach space $E$ is denoted by $i d_{E}$. Given an operator ideal $\mathcal{I}$ and a Banach space $F$, it is clear that

$$
i d_{F} \in \mathcal{I}(F ; F) \Longleftrightarrow \mathcal{I}(E ; F)=\mathcal{L}(E ; F) \text { for every } E \text {. }
$$

Lemma 3.4. Let $\mathcal{I}_{1}, \mathcal{I}_{2}, \mathcal{I}$ be operator ideals, $n \in \mathbb{N}$ and $E, E_{1}, \ldots, E_{n}$, $F$ be Banach spaces.

(a) If $\mathcal{I}_{1} \circ \mathcal{L}\left(E_{1}, \ldots, E_{n} ; F\right) \subseteq \mathcal{I}_{2} \circ \mathcal{L}\left(E_{1}, \ldots, E_{n} ; F\right)$, then $\mathcal{I}_{1}\left(E_{j} ; F\right) \subseteq \mathcal{I}_{2}\left(E_{j} ; F\right)$ for every $j=1, \ldots, n$. In particular, If $\mathcal{I} \circ \mathcal{L}\left(E_{1}, \ldots, E_{n} ; F\right)=\mathcal{L}\left(E_{1}, \ldots, E_{n}\right.$; $F)$, then $\mathcal{I}\left(E_{j} ; F\right)=\mathcal{L}\left(E_{j} ; F\right)$ for every $j=1, \ldots, n$.

(b) If $\mathcal{I}_{1} \circ \mathcal{P}\left({ }^{n} E ; F\right) \subseteq \mathcal{I}_{2} \circ \mathcal{P}\left({ }^{n} E ; F\right)$, then $\mathcal{I}_{1}(E ; F) \subseteq \mathcal{I}_{2}(E ; F)$. In particular, if $\mathcal{I} \circ \mathcal{P}\left({ }^{n} E ; F\right)=\mathcal{P}\left({ }^{n} E ; F\right)$, then $\mathcal{I}(E ; F)=\mathcal{L}(E ; F)$.

Proof. (a) Let $v \in \mathcal{I}_{1}\left(E_{j} ; F\right)$. For $i \neq j$, fix $0 \neq a_{i} \in E_{i}, \varphi_{i} \in\left(E_{i}\right)^{\prime}$ with $\varphi_{i}\left(a_{i}\right)=1$ and define $A \in \mathcal{L}\left(E_{1}, \ldots, E_{n} ; F\right)$ by

$$
A\left(x_{1}, \ldots, x_{n}\right)=\varphi_{1}\left(x_{1}\right) \cdots \varphi_{j-1}\left(x_{j-1}\right) \varphi_{j+1}\left(x_{j+1}\right) \cdots \varphi_{n}\left(x_{n}\right) v\left(x_{j}\right) .
$$


Letting $C\left(x_{1}, \ldots, x_{n}\right)=\varphi_{1}\left(x_{1}\right) \cdots \varphi_{j-1}\left(x_{j-1}\right) \varphi_{j+1}\left(x_{j+1}\right) \cdots \varphi_{n}\left(x_{n}\right) x_{j}$ we have that $C \in \mathcal{L}\left(E_{1}, \ldots, E_{n} ; E_{j}\right)$ and $A=v \circ C$. Hence $A \in \mathcal{I}_{1} \circ \mathcal{L}\left(E_{1}, \ldots, E_{n} ; F\right)$, so by assumption $A \in \mathcal{I}_{2} \circ \mathcal{L}\left(E_{1}, \ldots, E_{n} ; F\right)$. Let $A=u \circ B$, where $u \in \mathcal{I}_{2}(G ; F)$ and $B \in \mathcal{L}\left(E_{1}, \ldots, E_{n} ; G\right)$. For every $x \in E_{j}$,

$$
\begin{aligned}
v(x) & =A\left(a_{1}, \ldots, a_{j-1}, x, a_{j+1}, \ldots, a_{n}\right) \\
& =\left(u \circ B\left(a_{1}, \ldots, a_{j-1}, \cdot, a_{j+1}, \ldots, a_{n}\right)\right)(x),
\end{aligned}
$$

hence we find that $v=u \circ B\left(a_{1}, \ldots, a_{j-1}, \cdot, a_{j+1}, \ldots, a_{n}\right)$, so $v \in \mathcal{I}_{2}\left(E_{j} ; F\right)$ as $u$ belongs to $\mathcal{I}_{2}$.

(b) Given $v \in \mathcal{I}_{1}(E ; F)$, fix $0 \neq a \in E$ and $\varphi \in E^{\prime}$ with $\varphi(a)=1$ and define $P \in \mathcal{P}\left({ }^{n} E ; F\right)$ by $P(x)=\varphi(x)^{n-1} v(x)$. Letting $R(x)=\varphi(x)^{n-1} x$ we have that $R \in \mathcal{P}\left({ }^{n} E ; E\right)$ and $P=v \circ R$. Hence $P \in \mathcal{I}_{1} \circ \mathcal{P}\left({ }^{n} E ; F\right)$, so by assumption $P \in \mathcal{I}_{2} \circ \mathcal{P}\left({ }^{n} E ; F\right)$. Let $P=u \circ Q$, where $u \in \mathcal{I}_{2}(G ; F)$ and $Q \in \mathcal{P}\left({ }^{n} E ; G\right)$. Since $\check{P}=u \circ \check{Q}$, for every $x \in E$,

$$
\begin{aligned}
(u \circ \check{Q}(a, \ldots, a, \cdot))(x) & =u(\check{Q}(a, \ldots, a, x))=\check{P}(a, \ldots, a, x) \\
& =\frac{1}{n}(v(x)+(n-1) \varphi(x) v(a)) .
\end{aligned}
$$

Therefore, $n(u \circ \check{Q}(a, \ldots, a, \cdot))=v+(n-1) \varphi(\cdot) v(a)$. It follows that $v \in \mathcal{I}_{2}(E ; F)$ as $u$ belongs to $\mathcal{I}_{2}$ and $\varphi(\cdot) v(a)$ is a finite rank operator.

In Remark 4.4 we shall see that the converse of Lemma 3.4 does not hold.

Proposition 3.5. $\quad$ Let $\mathcal{I}$ be an operator ideal and $F$ be a Banach space. The following are equivalent:

(a) $i d_{F} \in \mathcal{I}(F ; F)$.

(b) $\mathcal{I} \circ \mathcal{L}\left(E_{1}, \ldots, E_{n} ; F\right)=\mathcal{L}\left(E_{1}, \ldots, E_{n} ; F\right)$ for every $n$ and every $E_{1}, \ldots, E_{n}$.

(c) $\mathcal{I} \circ \mathcal{L}\left(E_{1}, \ldots, E_{n} ; F\right)=\mathcal{L}\left(E_{1}, \ldots, E_{n} ; F\right)$ for some $n$ and every $E_{1}, \ldots, E_{n}$.

(d) $\mathcal{I} \circ \mathcal{P}\left({ }^{n} E ; F\right)=\mathcal{P}\left({ }^{n} E ; F\right)$ for every $n$ and every $E$.

(e) $\mathcal{I} \circ \mathcal{P}\left({ }^{n} E ; F\right)=\mathcal{P}\left({ }^{n} E ; F\right)$ for some $n$ and every $E$.

Proof. It is obvious that (a) implies all the others. (b) $\Longrightarrow(\mathrm{c})$ and (d) $\Longrightarrow(\mathrm{e})$ are obvious too. (c) $\Longrightarrow$ (a) and (e) $\Longrightarrow$ (a) follow from Lemma 3.4 (a) and (b), respectively.

Let $\mathcal{I}$ be a closed operator ideal. By Proposition 3.3, $\mathcal{I} \circ \mathcal{L}$ and $\mathcal{I} \circ \mathcal{P}$ become Banach multi and polynomial ideals, respectively, with respect to the usual sup norm. For arbitrary ideals, we proceed as follows. 
Definition 3.6. $\quad$ Let $\mathcal{I}$ be a normed operator ideal.

(a) For $A \in \mathcal{I} \circ \mathcal{L}\left(E_{1}, \ldots, E_{n} ; F\right)$, define

$$
\|A\|_{\mathcal{I} \circ \mathcal{L}}:=\inf \left\{\|u\|_{\mathcal{I}}\|B\|: A=u \circ B, B \in \mathcal{L}\left(E_{1}, \ldots, E_{n} ; G\right), u \in \mathcal{I}(G ; F)\right\} .
$$

(b) For $P \in \mathcal{I} \circ \mathcal{P}\left({ }^{n} E ; F\right)$, define $\|P\|_{\mathcal{I} \circ \mathcal{P}, 1}:=\|\check{P}\|_{\mathcal{I} \circ \mathcal{L}}$ and

$$
\|P\|_{\mathcal{I} \circ \mathcal{P}, 2}:=\inf \left\{\|u\|_{\mathcal{I}}\|Q\|: P=u \circ Q, Q \in \mathcal{P}\left({ }^{n} E ; G\right), u \in \mathcal{I}(G ; F)\right\} .
$$

Proposition 3.7. $\quad$ Let $\mathcal{I}$ be a normed (Banach) operator ideal.

(a) $\|\cdot\|_{\mathcal{I} \circ \mathcal{L}}$ makes $\mathcal{I} \circ \mathcal{L}$ a normed $\left(\right.$ Banach) multi-ideal. Moreover, $\|A\|_{\mathcal{I} \circ \mathcal{L}}=$ $\left\|A_{L}\right\|_{\mathcal{I}}$ for every $A \in \mathcal{I} \circ \mathcal{L}\left(E_{1}, \ldots, E_{n} ; F\right)$.

(b) Both $\|\cdot\|_{\mathcal{I} \circ \mathcal{P}, 1}$ and $\|\cdot\|_{\mathcal{I} \circ \mathcal{P}, 2}$ make $\mathcal{I} \circ \mathcal{P}$ a normed (Banach) polynomial ideal. Moreover, for every $P \in \mathcal{I} \circ \mathcal{P}\left({ }^{n} E ; F\right)$,

$$
\begin{gathered}
\|P\|_{\mathcal{I} \circ \mathcal{P}, 1}=\left\|P_{L}\right\|_{\mathcal{I}}=\inf \left\{\|A\|_{\mathcal{I} \circ \mathcal{L}}: A \in \mathcal{I} \circ \mathcal{L}\left({ }^{n} E ; F\right) \text { and } \hat{A}=P\right\}, \\
\|P\|_{\mathcal{I} \circ \mathcal{P}, 2}=\left\|P_{L, s}\right\|_{\mathcal{I}} \text { and } \\
\|P\|_{\mathcal{I}^{\circ} \mathcal{P}, 2} \leq\|P\|_{\mathcal{I} \circ \mathcal{P}, 1} \leq c(n, E)\|P\|_{\mathcal{I} \circ \mathcal{P}, 2} .
\end{gathered}
$$

Proof. It is also folklore that $\|\cdot\|_{\mathcal{I} \circ \mathcal{L}}$ is a multi-ideal (complete) norm and that $\|\cdot\|_{\mathcal{I} \circ \mathcal{P}, 1}$ and $\|\cdot\|_{\mathcal{I} \circ \mathcal{P}, 2}$ are polynomial ideal (complete) norms. We keep the notation introduced in the proof of Proposition 3.2. Let $A \in \mathcal{I}$ 。 $\mathcal{L}\left(E_{1}, \ldots, E_{n} ; F\right)$. If $A=u \circ B$ with $B \in \mathcal{L}\left(E_{1}, \ldots, E_{n} ; G\right)$ and $u \in \mathcal{I}(G ; F)$,

$$
\left\|A_{L}\right\|_{\mathcal{I}}=\left\|(u \circ B)_{L}\right\|_{\mathcal{I}}=\left\|u \circ B_{L}\right\|_{\mathcal{I}} \leq\|u\|_{\mathcal{I}}\left\|B_{L}\right\|=\|u\|_{\mathcal{I}}\|B\| .
$$

Taking the infimum over all such factorizations we have that $\left\|A_{L}\right\|_{\mathcal{I}} \leq\|A\|_{\mathcal{I} \circ \mathcal{L} \text {. }}$ It follows that $\left\|A_{L}\right\|_{\mathcal{I}}=\|A\|_{\mathcal{I}_{\circ} \mathcal{L}}$ because $A=A_{L} \circ \sigma_{n}$ and $\left\|\sigma_{n}\right\|=1$. Let $P \in \mathcal{I} \circ \mathcal{P}\left({ }^{n} E ; F\right)$ and let $i_{n}: \widehat{\otimes}_{\pi}^{n, s} E \longrightarrow \widehat{\otimes}_{\pi}^{n} E$ be the formal inclusion. Since $\check{P}=P_{L} \circ S_{n} \circ \sigma_{n}$, we have that $\check{P}_{L}=P_{L} \circ S_{n}$. By $[19$, p. 162] we know that $\left\|S_{n}\right\|=1$, so

$$
\begin{aligned}
\|P\|_{\mathcal{I} \circ \mathcal{P}, 1} & =\|\check{P}\|_{\mathcal{I} \circ \mathcal{L}}=\left\|\check{P}_{L}\right\|_{\mathcal{I}}=\left\|P_{L} \circ S_{n}\right\|_{\mathcal{I}} \leq\left\|P_{L}\right\|_{\mathcal{I}}\left\|S_{n}\right\|=\left\|P_{L}\right\|_{\mathcal{I}} \\
& =\left\|\check{P}_{L} \circ i_{n}\right\|_{\mathcal{I}} \leq\left\|\check{P}_{L}\right\|_{\mathcal{I}}\left\|i_{n}\right\|=\left\|\check{P}_{L}\right\|_{\mathcal{I}}=\|\check{P}\|_{\mathcal{I} \circ \mathcal{L}}=\|P\|_{\mathcal{I} \circ \mathcal{P}, 1} .
\end{aligned}
$$

Now let $A \in \mathcal{I} \circ \mathcal{L}\left({ }^{n} E ; F\right)$ be such that $\hat{A}=P$. For every $\sigma \in \Delta_{n}$, consider the $n$-linear mappings

$$
\begin{gathered}
B^{\sigma}: E \times \cdots \times E \longrightarrow \widehat{\otimes}_{\pi}^{n} E ; B^{\sigma}\left(x_{1}, \ldots, x_{n}\right)=x_{\sigma(1)} \otimes \cdots \otimes x_{\sigma(n)} . \\
A_{\sigma}: E \times \cdots \times E \longrightarrow F ; A_{\sigma}\left(x_{1}, \ldots, x_{n}\right)=A\left(x_{\sigma(1)}, \ldots, x_{\sigma(n)}\right) .
\end{gathered}
$$


It is easy to check that $\left(A_{\sigma}\right)_{L}=A_{L} \circ\left(B^{\sigma}\right)_{L}$, so, for every $\sigma \in \Delta_{n},\left(A_{\sigma}\right)_{L}$ belongs to $\mathcal{I}$ and

$$
\left\|\left(A_{\sigma}\right)_{L}\right\|_{\mathcal{I}} \leq\left\|A_{L}\right\|_{\mathcal{I}}\left\|\left(B^{\sigma}\right)_{L}\right\|=\left\|A_{L}\right\|_{\mathcal{I}}\left\|B^{\sigma}\right\|=\left\|A_{L}\right\|_{\mathcal{I}}
$$

From $\check{P}=A_{S}=\frac{1}{n !} \sum_{\sigma \in \Delta_{n}} A_{\sigma}$ we have

$$
\begin{aligned}
\|P\|_{\mathcal{I} \circ \mathcal{P}, 1} & =\|\check{P}\|_{\mathcal{I} \circ \mathcal{L}}=\left\|\frac{1}{n !} \sum_{\sigma \in \Delta_{n}} A_{\sigma}\right\|_{\mathcal{I}_{\circ} \mathcal{L}} \leq \frac{1}{n !} \sum_{\sigma \in \Delta_{n}}\left\|A_{\sigma}\right\|_{\mathcal{I}_{\circ} \mathcal{L}} \\
& =\frac{1}{n !} \sum_{\sigma \in \Delta_{n}}\left\|\left(A_{\sigma}\right)_{L}\right\|_{\mathcal{I}} \leq \frac{1}{n !} \sum_{\sigma \in \Delta_{n}}\left\|A_{L}\right\|_{\mathcal{I}}=\left\|A_{L}\right\|_{\mathcal{I}}=\|A\|_{\mathcal{I} \circ \mathcal{L}},
\end{aligned}
$$

for every $A \in \mathcal{I} \circ \mathcal{L}\left({ }^{n} E ; F\right)$ such that $\hat{A}=P$. Therefore,

$$
\|P\|_{\mathcal{I} \circ \mathcal{P}, 1} \leq \inf \left\{\|A\|_{\mathcal{I} \circ \mathcal{L}}: A \in \mathcal{I} \circ \mathcal{L}\left({ }^{n} E ; F\right) \text { and } \hat{A}=P\right\} .
$$

The reverse inequality is obvious as $\check{P} \in \mathcal{I} \circ \mathcal{L}\left({ }^{n} E ; F\right)$ and $\widehat{\ddot{P}}=P$. The argument used to prove that $\left\|A_{L}\right\|_{\mathcal{I}}=\|A\|_{\mathcal{I}_{0} \mathcal{L}}$ can be repeated to prove that $\left\|P_{L, s}\right\|_{\mathcal{I}}=\|P\|_{\mathcal{I} \circ \mathcal{P}, 2}$. The estimates $\|P\|_{\mathcal{I} \circ \mathcal{P}, 2} \leq\|P\|_{\mathcal{I} \circ \mathcal{P}, 1} \leq c(n, E)\|P\|_{\mathcal{I} \circ \mathcal{P}, 2}$ follow easily from the ideal property, the already proved identities $\|P\|_{\mathcal{I}_{\circ} \mathcal{P}, 1}=$ $\left\|P_{L}\right\|_{\mathcal{I}},\|P\|_{\mathcal{I} \circ \mathcal{P}, 2}=\left\|P_{L, s}\right\|_{\mathcal{I}}$ and the already mentioned inequalities $\pi \leq \pi_{s} \leq$ $c(n, E) \pi$.

Sometimes these new norms coincide with the usual sup norms:

Corollary 3.8. If either

(a) $\mathcal{I}$ is a closed operator ideal, $A \in \mathcal{I} \circ \mathcal{L}\left(E_{1}, \ldots, E_{n} ; F\right)$ and $P \in \mathcal{I} \circ \mathcal{P}\left({ }^{n} E ; F\right)$, or

(b) $\mathcal{I}$ is an arbitrary normed operator ideal, $A \in \mathcal{L}\left(E_{1}, \ldots, E_{n}\right)$ and $P \in \mathcal{P}\left({ }^{n} E\right)$, then $\|A\|_{\mathcal{I} \circ \mathcal{L}}=\|A\|,\|P\|_{\mathcal{I} \circ \mathcal{P}, 1}=\|\check{P}\|$ and $\|P\|_{\mathcal{I} \circ \mathcal{P}, 2}=\|P\|$.

Proof. In both cases, Proposition 3.7 gives

$$
\begin{gathered}
\|A\|_{\mathcal{I} \circ \mathcal{L}}=\left\|A_{L}\right\|_{\mathcal{I}}=\left\|A_{L}\right\|=\|A\|, \\
\|P\|_{\mathcal{I} \circ \mathcal{P}, 1}=\left\|P_{L}\right\|_{\mathcal{I}}=\left\|P_{L}\right\|=\|\check{P}\|, \\
\|P\|_{\mathcal{I} \circ \mathcal{P}, 2}=\left\|P_{L, s}\right\|_{\mathcal{I}}=\left\|P_{L, s}\right\|=\|P\| .
\end{gathered}
$$




\section{$\S 4$. Strictly Singular Polynomials}

An operator $u \in \mathcal{L}(E ; F)$ is strictly singular, in symbols $u \in S S(E ; F)$, if for every infinite-dimensional subspace $G$ of $E$, the restriction of $u$ to $G$, $\left.u\right|_{G}: G \rightarrow u(G)$, is not an isomorphism; or, equivalently, if for every infinitedimensional subspace $G$ of $E$ and every $\varepsilon>0$, there is $x \in G$ such that $\|u(x)\|<\varepsilon\|x\|$.

For the closed ideals $\mathcal{K}$ and $\mathcal{W}$ we have already mentioned that a polynomial $P$ is compact (weakly compact) if and only if $P \in \mathcal{K} \circ \mathcal{P}(P \in \mathcal{W} \circ \mathcal{P})$. Since the ideal of all strictly singular linear operators, denoted by $S S$, is closed, the following definition is quite natural:

Definition 4.1. Let $P \in \mathcal{P}\left({ }^{n} E ; F\right)$. We say that $P$ is strictly singular if $P \in S S \circ \mathcal{P}\left({ }^{n} E ; F\right)$.

Examples 4.2 (Strictly singular polynomials). It is plain that every scalar-valued continuous homogeneous polynomial is strictly singular. More generally we have that every compact homogeneous polynomial is strictly singular: $\mathcal{P}_{\mathcal{K}}=\mathcal{K} \circ \mathcal{P} \subseteq S S \circ \mathcal{P}$ because compact linear operators are strictly singular. In particular, every homogeneous polynomial from $c_{0}$ to $\ell_{p}, 1 \leq p<+\infty$, is strictly singular ([3, p. 216]); and, for $n q<p$, every $n$-homogeneous polynomial from $\ell_{p}$ to $\ell_{q}$ is strictly singular ([1, Theorem 4.2]). The existence of non-compact strictly singular polynomials is an easy consequence of Corollary 4.6 .

Examples 4.3 (Non-strictly singular polynomials).

(a) Consider the bilinear mapping

$$
A: \ell_{2} \times \ell_{2} \longrightarrow \ell_{1} ; A\left(\left(x_{j}\right),\left(y_{j}\right)\right)=\left(x_{j} y_{j}\right)
$$

Let $\left(e_{j}\right)$ be the standard unit vectors of $\ell_{2}$, and let $D$ be the closed span of the diagonal vectors $e_{j} \otimes e_{j}$ in $\ell_{2} \widehat{\otimes}_{\pi} \ell_{2}$. By [28, Example 2.10], extending to $\ell_{1}$ the linear operator

$$
\left(\lambda_{1}, \ldots, \lambda_{k}, 0,0, \ldots,\right) \mapsto I\left(\left(\lambda_{1}, \ldots, \lambda_{k}, 0,0, \ldots,\right)\right):=\sum_{j=1}^{k} \lambda_{j} e_{j} \otimes e_{j}
$$

we obtain an isometric isomorphism $I: \ell_{1} \longrightarrow D$. For every $x=\sum_{j=1}^{k} \lambda_{j} e_{j} \otimes$ 
$e_{j} \in D$

$$
\begin{aligned}
\left(I \circ A_{L}\right)(x) & =I\left(A_{L}\left(\sum_{j=1}^{k} \lambda_{j} e_{j} \otimes e_{j}\right)\right)=I\left(\sum_{j=1}^{k} \lambda_{j} A_{L}\left(e_{j} \otimes e_{j}\right)\right) \\
& =I\left(\sum_{j=1}^{k} \lambda_{j} A\left(e_{j}, e_{j}\right)\right)=I\left(\sum_{j=1}^{k} \lambda_{j} e_{j}\right) \\
& =\sum_{j=1}^{k} \lambda_{j} e_{j} \otimes e_{j}=x,
\end{aligned}
$$

showing that $\left.I \circ A_{L}\right|_{D}$ is the identity on $D$, hence an isomorphism. Therefore, $\left.A_{L}\right|_{D}=\left.I^{-1} \circ I \circ A_{L}\right|_{D}$ is an isomorphism as well, proving that $A_{L}$ is not strictly singular. So, $A \notin S S \circ \mathcal{L}\left({ }^{2} \ell_{2} ; \ell_{1}\right)$. By Proposition 3.2 it follows that $\hat{A} \notin S S \circ \mathcal{P}\left({ }^{2} \ell_{2} ; \ell_{1}\right)$.

(b) Given $n \in \mathbb{N}$ and an infinite-dimensional Banach space $E$, consider the canonical polynomial $\delta_{n} \in \mathcal{P}\left({ }^{n} E ; \widehat{\otimes}_{\pi}^{n, s} E\right)$ defined in the proof of Proposition 3.2. It is clear that $\left(\delta_{n}\right)_{L}$ is the identity operator on $\widehat{\otimes}_{\pi}^{n, s} E$, hence not strictly singular as the identity operator on an infinite-dimensional space is never strictly singular. Therefore, $\delta_{n} \notin S S \circ \mathcal{P}\left({ }^{n} E ; \widehat{\otimes}_{\pi}^{n, s} E\right)$.

Remark 4.4. Since $\ell_{1}$ and $\ell_{2}$ do not have isomorphic infinite-dimensional closed subspaces, $S S\left(\ell_{2} ; \ell_{1}\right)=\mathcal{L}\left(\ell_{2} ; \ell_{1}\right)$. By Example $4.3($ a) we know that $S S \circ$ $\mathcal{P}\left({ }^{2} \ell_{2} ; \ell_{1}\right) \neq \mathcal{P}\left({ }^{2} \ell_{2} ; \ell_{1}\right)$ (therefore $\left.S S \circ \mathcal{L}\left({ }^{2} \ell_{2} ; \ell_{1}\right) \neq \mathcal{L}\left({ }^{2} \ell_{2} ; \ell_{1}\right)\right)$, so the converse of Lemma 3.4 does not hold true.

Recall that the Banach spaces $E$ and $F$ are called totally incomparable if they do not have isomorphic infinite-dimensional closed subspaces. It is well known that any two different spaces of the family $\left\{c_{0}, \ell_{p}, 1 \leq p<+\infty\right\}$ are totally incomparable.

Proposition 4.5. Let $n \in \mathbb{N}$ and $E$ and $F$ be Banach spaces such that $\widehat{\otimes}_{\pi}^{n, s} E$ and $F$ are totally incomparable. Then $\mathcal{P}\left({ }^{n} E ; F\right)=S S \circ \mathcal{P}\left({ }^{n} E ; F\right)$.

Proof. Given $P \in \mathcal{P}\left({ }^{n} E ; F\right), P_{L} \in \mathcal{L}\left(\widehat{\otimes}_{\pi}^{n, s} E ; F\right)$, which is strictly singular by assumption.

Corollary 4.6. $\mathcal{P}\left({ }^{n} \ell_{1} ; \ell_{p}\right)=S S \circ \mathcal{P}\left({ }^{n} \ell_{1} ; \ell_{p}\right)$ and $\mathcal{P}\left({ }^{n} \ell_{1} ; c_{0}\right)=S S \circ$ $\mathcal{P}\left({ }^{n} \ell_{1} ; c_{0}\right)$ for every $n$ and every $1<p<+\infty$. 
Proof. By [28, Exercise 2.6] we know that, for every $n, \widehat{\otimes}_{\pi}^{n} \ell_{1}$ is isometrically isomorphic to $\ell_{1}$. So $\widehat{\otimes}_{\pi}^{n} \ell_{1}$ and $\ell_{p}, 1<p<+\infty$ (or $c_{0}$ ) are totally incomparable. The result follows from Proposition 4.5.

By Proposition 3.5 we know that Corollary 4.6 is no longer true for $p=1$. Besides being a partial converse of Proposition 4.5, the next result provides nice consequences on the existence of non-strictly singular polynomials.

Proposition 4.7. Let $n \in \mathbb{N}$ and $E$ and $F$ be Banach spaces. If $\mathcal{P}\left({ }^{n} E\right.$; $F)=S S \circ \mathcal{P}\left({ }^{n} E ; F\right)$, then no infinite-dimensional subspace of $F$ is isomorphic to any complemented subspace of $\widehat{\otimes}_{\pi}^{k, s} E, k=1, \ldots, n$.

Proof. Since $\widehat{\otimes}_{\pi}^{k, s} E$ is a complemented subspace of $\widehat{\otimes}_{\pi}^{n, s} E[4$, Corollary $4]$, it suffices to show that no infinite-dimensional subspace of $F$ is isomorphic to any complemented subspace of $\widehat{\otimes}_{\pi}^{n, s} E$. Assume that there exist an infinitedimensional subspace $X$ of $F$, a complemented subspace $Y$ of $\widehat{\otimes}_{\pi}^{n, s} E$ and an onto isomorphism $u: Y \longrightarrow X$. Let $p_{Y}: \widehat{\otimes}_{\pi}^{n, s} E \longrightarrow Y$ be a projection onto $Y$. Consider the chain

$$
E \stackrel{\delta_{n}}{\longrightarrow} \widehat{\otimes}_{\pi}^{n, s} E \stackrel{p_{Y}}{\longrightarrow} Y \stackrel{u}{\longrightarrow} X \stackrel{i_{X}}{\longrightarrow} F
$$

where $\delta_{n}$ is the canonical polynomial defined in the proof of Proposition 3.2 and $i_{X}: X \longrightarrow F$ is the formal inclusion. Defining $Q:=i_{X} \circ u \circ p_{Y} \circ \delta_{n} \in \mathcal{P}\left({ }^{n} E ; F\right)$ it follows that $Q_{L}=i_{X} \circ u \circ p_{Y}$, which is not strictly singular because $\left.i_{X} \circ u \circ p_{Y}\right|_{Y}$ is an isomorphism onto its range. Thus $Q \notin S S \circ \mathcal{P}\left({ }^{n} E ; F\right)$.

\section{Corollary 4.8.}

(a) $\mathcal{P}\left({ }^{n} \ell_{n p} ; \ell_{p}\right) \neq S S \circ \mathcal{P}\left({ }^{n} \ell_{n p} ; \ell_{p}\right)$ for every $n \in \mathbb{N}$ and every $1 \leq p<+\infty$.

(b) Let $1 \leq p<+\infty$ and let $E$ be such that $\ell_{p}$ is a quotient of $E$. Then $\mathcal{P}\left({ }^{n} E ; \ell_{1}\right) \neq S S \circ \mathcal{P}\left({ }^{n} E ; \ell_{1}\right)$ for every $n \geq p$.

Proof. (a) Since $\ell_{p}$ is a complemented subspace of $\widehat{\otimes}_{\pi}^{n, s} \ell_{n p}$ (see [5, Theorem 13]), the result follows from Proposition 4.7. The case $p=1$ also follows from an easy adaptation of [28, Example 2.10] and Example 4.3(a). Actually, the polynomial $P\left(\left(x_{j}\right)_{j=1}^{\infty}\right)=\left(x_{j}^{n}\right)_{j=1}^{\infty}$ does not belong to $S S \circ \mathcal{P}\left({ }^{n} \ell_{n} ; \ell_{1}\right)$.

(b) For $n \geq p, \ell_{\infty}$ is a subspace of $\mathcal{P}\left({ }^{n} E\right)=\left(\widehat{\otimes}_{\pi_{s}}^{n, s} E\right)^{\prime}$ by [18, Corollary 1.56]. So by [22, Proposition I.2.e.8] it follows that $\ell_{1}$ is a complemented subspace of $\widehat{\otimes}_{\pi_{s}}^{n, s} E$. The result follows again from Proposition 4.7 as $\widehat{\otimes}_{\pi}^{n, s} E$ and $\widehat{\otimes}_{\pi_{s}}^{n, s} E$ are isomorphic. 


\section{§5. Absolutely Summing Multilinear Mappings}

Let $\Pi(E ; F)$ denote the space of all absolutely summing linear operators from $E$ to $F$. In this section we shall establish relationships between the multiideal $\Pi \circ \mathcal{L}$ and other three already investigated classes of multilinear mappings which generalize the absolutely summing linear operators.

Definition 5.1. An $n$-linear mapping $A \in \mathcal{L}\left(E_{1}, \ldots, E_{n} ; F\right)$ is said to be

- semi-integral $[2,7,13]$ if there are $C \geq 0$ and a regular Borel probability measure $\mu$ on $B_{E_{1}^{\prime}} \times \cdots \times B_{E_{n}^{\prime}}$ such that for every $\left(x_{1}, \ldots, x_{n}\right) \in E_{1} \times \cdots \times E_{n}$,

$$
\left\|A\left(x_{1}, \ldots, x_{n}\right)\right\| \leq C \cdot \int_{B_{E_{1}^{\prime}} \times \cdots \times B_{E_{n}^{\prime}}}\left|\varphi_{1}\left(x_{1}\right) \cdots \varphi_{n}\left(x_{n}\right)\right| d \mu\left(\varphi_{1}, \ldots, \varphi_{n}\right) .
$$

- dominated $[6,7,8,10,24]$ if $\left(A\left(x_{j}^{1}, \ldots, x_{j}^{n}\right)\right)_{j=1}^{\infty}$ is absolutely $\frac{1}{n}$-summable in $F$ whenever $\left(x_{j}^{k}\right)_{j=1}^{\infty}$ are weakly summable in $E_{k}, k=1, \ldots, n$.

- strongly summing $[11,17]$ if there is $C \geq 0$ such that for every $k \in \mathbb{N}$ and every $x_{1}^{i}, \ldots, x_{k}^{i} \in E_{i}, i=1, \ldots, n$,

$$
\sum_{j=1}^{k}\left\|A\left(x_{j}^{1}, \ldots, x_{j}^{n}\right)\right\| \leq C \cdot \sup _{T \in B_{\mathcal{L}\left(E_{1}, \ldots, E_{n}\right)}} \sum_{j=1}^{k}\left|T\left(x_{j}^{1}, \ldots, x_{j}^{n}\right)\right| .
$$

Strongly summing multilinear mappings were introduced by V. Dimant [17] for real Banach spaces, but complex scalars work for our purposes as well. The spaces of all semi-integral, dominated and strongly summing $n$-linear mappings from $E_{1} \times \cdots \times E_{n}$ to $F$ are denoted by $\mathcal{L}_{s i}\left(E_{1}, \ldots, E_{n} ; F\right), \mathcal{L}_{d}\left(E_{1}, \ldots, E_{n} ; F\right)$ and $\mathcal{L}_{s s}\left(E_{1}, \ldots, E_{n} ; F\right)$, respectively. These spaces become Banach spaces with the semi-integral, dominated and strongly summing norms, which definitions can be found in [2], [24] and [17], respectively.

R. Alencar-M. C. Matos [2] introduced a reasonable crossnorm $\sigma$ such that $\mathcal{L}_{s i}\left(E_{1}, \ldots, E_{n}\right)$ is isometrically isomorphic to $\left(E_{1} \otimes_{\sigma} \cdots \otimes_{\sigma} E_{n}\right)^{\prime}[2$, Theorem 4.8]. When we consider the linearization $A_{L}$ of a semi-integral mapping $A \in$ $\mathcal{L}_{s i}\left(E_{1}, \ldots, E_{n} ; F\right)$ defined on $E_{1} \widehat{\otimes}_{\sigma} \cdots \widehat{\otimes}_{\sigma} E_{n}$, it will be denoted $A_{L}^{\sigma}$. As to vector-valued mappings we have:

\section{Theorem 5.2.}

(a) $\left(\left[2\right.\right.$, Proposition 4.10]) Let $A \in \mathcal{L}\left(E_{1}, \ldots, E_{n} ; F\right)$. If $A_{L}^{\sigma}: E_{1} \widehat{\otimes}_{\sigma} \cdots \widehat{\otimes}_{\sigma} E_{n}$

$\longrightarrow F$ is absolutely summing, then $A$ is semi-integral.

(b) ([2, Corollaries 5.8 and 5.9]) Let $K_{1}, \ldots, K_{n}$ be compact Hausdorff spaces, 
$F$ be an arbitrary Banach space and $A \in \mathcal{L}\left(C\left(K_{1}\right), \ldots, C\left(K_{n}\right) ; F\right)$. Then, $A$ is semi-integral if and only if $A_{L}^{\sigma}: C\left(K_{1}\right) \widehat{\otimes}_{\sigma} \cdots \widehat{\otimes}_{\sigma} C\left(K_{n}\right) \longrightarrow F$ is absolutely summing.

The next five results show that, like the operator ideal $\Pi$, the multi-ideal П॰ $\mathcal{L}$ is neither very small nor very large. We begin with the following combination of Lemma 3.4 with the Weak Dvoretzky-Rogers Theorem [16, Theorem 2.18]:

Proposition 5.3 (Dvoretzky-Rogers-type theorem). The following assertions are equivalent for a Banach space $E$ :

(a) $\Pi \circ \mathcal{L}\left({ }^{n} E ; E\right)=\mathcal{L}\left({ }^{n} E ; E\right)$ for every $n \in \mathbb{N}$.

(b) $\Pi \circ \mathcal{L}\left({ }^{n} E ; E\right)=\mathcal{L}\left({ }^{n} E ; E\right)$ for some $n \in \mathbb{N}$.

(c) $E$ is finite-dimensional.

Proposition 5.4 (Grothendieck-type theorem). $\quad \Pi \circ \mathcal{L}\left(E_{1}, \ldots, E_{n} ; F\right)$ $=\mathcal{L}\left(E_{1}, \ldots, E_{n} ; F\right)$ for every $n$, any $\mathcal{L}_{1}$-spaces $E_{1}, \ldots, E_{n}$ and any $\mathcal{L}_{2}$-space $F$.

Proof. Let $A \in \mathcal{L}\left(E_{1}, \ldots, E_{n} ; F\right)$. With the help of [14, Ex 23.17(c)] it is easy to see that $E_{1} \widehat{\otimes}_{\pi} \cdots \widehat{\otimes}_{\pi} E_{n}$ is an $\mathcal{L}_{1}$-space. So, $A_{L}$ is a linear operator from an $\mathcal{L}_{1}$-space into an $\mathcal{L}_{2}$-space, which is absolutely summing by Grothendieck's Theorem [16, Theorem 3.1].

Proposition 5.5 (Lindenstrauss-Pełczyński-type theorem). The following assertions are equivalent for an infinite-dimensional Banach space $E$ with unconditional basis.

(a) $\Pi \circ \mathcal{L}\left({ }^{n} E ; F\right)=\mathcal{L}\left({ }^{n} E ; F\right)$ for every $n \in \mathbb{N}$.

(b) $\Pi \circ \mathcal{L}\left({ }^{n} E ; F\right)=\mathcal{L}\left({ }^{n} E ; F\right)$ for some $n \in \mathbb{N}$.

(c) $E$ is isomorphic to $\ell_{1}(\Gamma)$ for some $\Gamma$ and $F$ is isomorphic to a Hilbert space.

Proof. Supposing (b), by Lemma 3.4 we find that $\Pi(E ; F)=\mathcal{L}(E ; F)$, so the Lindenstrauss-Pełczyński Theorem [23, Theorem 4.2] gives (c). The implication (c) $\Longrightarrow$ (a) follows from Proposition 5.4 and (a) $\Longrightarrow$ (b) is obvious.

By $\mathcal{L}_{\mathcal{W}}\left(E_{1}, \ldots, E_{n} ; F\right)$ we mean the closed subspace of $\mathcal{L}\left(E_{1}, \ldots, E_{n} ; F\right)$ formed by the weakly compact mappings and by $\mathcal{J}$ the ideal of all integral multilinear mappings (see, e.g., [12, Definition 2.1]).

Proposition 5.6. $\quad \mathcal{J} \subseteq \Pi \circ \mathcal{L} \subseteq\left(\mathcal{L}_{s s} \cap \mathcal{L}_{\mathcal{W}}\right)$. 
Proof. Let $A \in \mathcal{J}\left(E_{1}, \ldots, E_{n} ; F\right)$. By $\varepsilon$ we denote the injective norm and by $A_{L}^{\varepsilon}$ the linearization of $A$ defined on $E_{1} \widehat{\otimes}_{\varepsilon} \cdots \widehat{\otimes}_{\varepsilon} E_{n}$. By [12, Proposition 2.2 ] we know that $A_{L}^{\varepsilon}$ is integral, hence absolutely summing by [16, Proposition 5.5]. Consider the diagram

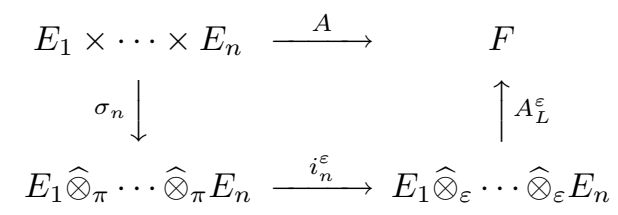

where $i_{n}^{\varepsilon}$ is the natural map. We know that $i_{n}^{\varepsilon}$ is continuous because $\varepsilon \leq \pi$ on $E_{1} \otimes \cdots \otimes E_{n}$. From $A_{L}=A_{L}^{\varepsilon} \circ i_{n}^{\varepsilon}$ it follows that $A_{L}$ is absolutely summing, so $A$ belongs to $\Pi \circ \mathcal{L}$. Now let $B \in \Pi \circ \mathcal{L}\left(E_{1}, \ldots, E_{n} ; F\right)$. Then $B=B_{L} \circ \sigma_{n}$, where $B_{L}$ is absolutely summing, hence weakly compact [16, Theorem 2.17]. The continuity of $\sigma_{n}$ and the weak compactness of $B_{L}$ yield that $B$ is weakly compact. $B$ is strongly summing by $[17$, p. 188].

Proposition 5.7. Let $K_{1}, \ldots, K_{n}$ be compact Hausdorff spaces. For $n$ linear mappings from $C\left(K_{1}\right) \times \cdots \times C\left(K_{n}\right)$ to an arbitrary Banach space, we have

$$
\mathcal{L}_{d} \subseteq \mathcal{L}_{s i} \subseteq \Pi \circ \mathcal{L} \subseteq\left(\mathcal{L}_{s s} \cap \mathcal{L}_{\mathcal{W}}\right)
$$

Proof. The first inclusion holds for multilinear mappings on arbitrary Banach spaces (see [7, Proposition 3.3(a)]). Given $A \in \mathcal{L}_{s i}\left(C\left(K_{1}\right), \ldots, C\left(K_{n}\right) ; F\right)$, $A_{L}=A_{L}^{\sigma} \circ i_{n}^{\sigma}$ where $i_{n}^{\sigma}: C\left(K_{1}\right) \widehat{\otimes}_{\pi} \cdots \widehat{\otimes}_{\pi} C\left(K_{n}\right) \longrightarrow C\left(K_{1}\right) \widehat{\otimes}_{\sigma} \cdots \widehat{\otimes}_{\sigma} C\left(K_{n}\right)$ is the natural map. $i_{n}^{\sigma}$ is continuous as $\sigma \leq \pi$ on $E_{1} \otimes \cdots \otimes E_{n}$ and $A_{L}^{\sigma}$ is absolutely summing by Theorem 5.2 , so $A_{L}$ is absolutely summing as well by the ideal property, that is $A \in \Pi \circ \mathcal{L}\left(C\left(K_{1}\right), \ldots, C\left(K_{n}\right) ; F\right)$.

A polynomial $P \in \mathcal{P}\left({ }^{n} E ; F\right)$ is dominated if $\left(P\left(x_{j}\right)\right)_{j=1}^{\infty}$ is absolutely $\frac{1}{n}$ summable in $F$ whenever $\left(x_{j}\right)_{j=1}^{\infty}$ is weakly summable in $E$. Contrary to the linear case, there are dominated polynomials which fail to be weakly compact [6, Proposition 46(d)]. But these polynomials are not defined on $C(K)$-spaces:

Corollary 5.8. Every dominated n-linear mapping from $C\left(K_{1}\right) \times \cdots \times$ $C\left(K_{n}\right)$ into an arbitrary Banach space is weakly compact. In particular, every dominated homogeneous polynomial from a $C(K)$-space into an arbitrary Banach space is weakly compact.

Proof. The multilinear case is immediate from Proposition 5.7. The polynomial case follows because a polynomial $P$ is dominated (weakly compact, 
respectively) if and only if $\check{P}$ is dominated (weakly compact, respectively) (see [6, Proposition 45] and [25, Proposition 3]).

Remark 5.9. (On a question of Alencar and Matos) In [2, Remark 4.11], the authors say that they do not know whether or not the converse of Theorem 5.2 (a) holds true (or, equivalently, whether or not the 'only if' part of Theorem 5.2(b) holds for semi-integral mappings on arbitrary Banach spaces). Suppose that the answer is yes. In this case, a repetition of the proof of Proposition 5.7 yields that the inclusions $\mathcal{L}_{d} \subseteq \mathcal{L}_{s i} \subseteq \Pi \circ \mathcal{L} \subseteq\left(\mathcal{L}_{s s} \cap \mathcal{L}_{\mathcal{W}}\right)$ hold true for multilinear mappings on arbitrary Banach spaces. But this is not the case as, reasoning as in the proof of Corollary 5.8, the existence of dominated nonweakly compact polynomials implies the existence of dominated non-weakly compact multilinear mappings. So the question is solved negatively.

\section{Acknowledgements}

Part of this paper was written while G.B. was a CNPq Postdoctoral Fellow in the Departamento de Análisis Matemático at Universidad de Valencia. He thanks Pilar Rueda and the members of the department for their kind hospitality. The authors thank the referee for his/her helpful suggestions.

\section{References}

[1] R. Alencar and K. Floret, Weak-strong continuity of multilinear mappings and the Pełczyński-Pitt theorem, J. Math. Anal. Appl. 206 (1997), no. 2, 532-546.

[2] R. Alencar and M. C. Matos, Some classes of multilinear mappings between Banach spaces, Publ. Dep. Análisis Matematico, Universidad Complutense de Madrid, Section 1, Number 12 (1989).

[3] R. M. Aron, Compact polynomials and compact differentiable mappings between Banach spaces, in Séminaire Pierre Lelong (Analyse), Année 1974/75, 213-222. Lecture Notes in Math., 524, Springer, Berlin.

[4] F. Blasco, Complementation in spaces of symmetric tensor products and polynomials, Studia Math. 123 (1997), no. 2, 165-173.

[5] Copies of $l_{p}$ in tensor products, Extracta Math. 15 (2000), no. 2, 283-290.

[6] G. Botelho, Ideals of polynomials generated by weakly compact operators, Note Mat. 25 (2005/06), no. 1, 69-102.

[7] G. Botelho and D. M. Pellegrino, Dominated polynomials on $\mathcal{L}_{p}$-spaces, Arch. Math. (Basel) 83 (2004), no. 4, 364-370.

[8] Scalar-valued dominated polynomials on Banach spaces, Proc. Amer. Math. Soc. 134 (2006), no. 6, 1743-1751 (electronic).

[9] , On symmetric ideals of multilinear mappings between Banach spaces, J. Aust. Math. Soc. 81 (2006), no. 1, 141-148.

[10] G. Botelho, D. Pellegrino and P. Rueda. Pietsch's factorization theorem for dominated polynomials, J. Funct. Anal. 243 (2007), 257-269.

[11] D. Carando and V. Dimant. On summability of bilinear operators, Math. Nachr. 259 (2003), 3-11. 
[12] R. Cilia, M. D'Anna and J. M. Gutiérrez, Polynomial characterization of $\mathcal{L}_{\infty}$-spaces, J. Math. Anal. Appl. 275 (2002), no. 2, 900-912.

[13] E. Çaliskan and D. Pellegrino, On multilinear extensions of absolutely summing operators, Rocky Mountain J. Math. 37 (2007), no. 4, 1137-1154.

[14] A. Defant and K. Floret, Tensor norms and operator ideals, North-Holland, Amsterdam, 1993.

[15] J. Diestel, H. Jarchow and A. Pietsch, Operator ideals, in Handbook of the geometry of Banach spaces, Vol. I, 437-496, North-Holland, Amsterdam, 2001.

[16] J. Diestel, H. Jarchow and A. Tonge, Absolutely summing operators, Cambridge Univ. Press, Cambridge, 1995.

[17] V. Dimant, Strongly $p$-summing multilinear operators, J. Math. Anal. Appl. 278 (2003), no. 1, 182-193.

[18] S. Dineen, Complex analysis on infinite-dimensional spaces, Springer, London, 1999.

[19] K. Floret, Natural norms on symmetric tensor products of normed spaces, Note Mat. 17 (1997), 153-188 (1999).

[20] Minimal ideals of $n$-homogeneous polynomials on Banach spaces, Results Math. 39 (2001), no. 3-4, 201-217.

[21] K. Floret and D. García, On ideals of polynomials and multilinear mappings between Banach spaces, Arch. Math. (Basel) 81 (2003), no. 3, 300-308.

[22] J. Lindenstrauss and L. Tzafriri, Classical Banach spaces. I, Springer, Berlin, 1977.

[23] J. Lindenstrauss and A. Pełczyński, Absolutely summing operators in $L_{p}$-spaces and their applications, Studia Math. 29 (1968), 275-326.

[24] M. C. Matos, Absolutely summing holomorphic mappings, An. Acad. Brasil. Ciênc. 68 (1996), no. 1, 1-13.

[25] A. Pełczyński, On weakly compact polynomial operators on $B$-spaces with DunfordPettis property, Bull. Acad. Polon. Sci. Sér. Sci. Math. Astronom. Phys. 11 (1963), 371-378.

[26] A. Pietsch, Ideals of multilinear functionals (designs of a theory), in Proceedings of the second international conference on operator algebras, ideals, and their applications in theoretical physics (Leipzig, 1983), 185-199, Teubner, Leipzig.

[27] R. Ryan, Applications of topological tensor products to infinite dimensional holomorphy, Thesis - Trinity College, 1980.

[28] - Introduction to tensor products of Banach spaces, Springer, London, 2002. 